\title{
MODELOS BAYESIANOS
}

Maria Emilia Camargo e Odorico Antōnio Bortoluzzi

Departamento de Estatîstica. Centro de Ciências Naturais e Exatas. UFSM. Santa Maria, RS.

\section{RESUMO}

Neste trabalho, são apresentados os conceitos básicos da Metodologia Bayesiana, bem como a utilização de uma sērie simulada para verificar a sensibilidade de mudanças de estado.

\section{SUMMARY}

CAMARGO, M.E. \& BORTOLUZZI, 0.A., 1990. Bayesian Models. Ciência e Natura, 11: 7-20, 1989.

In this work, will be presented the basic concept of Bayesian methodology, as well as the use of a simulated serie to check the sensibility of SBPC program to identify changes of state.

\section{INTRODUÇAO}

Um dos tōpicos da Estatīstica que tem merecido grande aten ção dos pesquisadores é a anālise de sēries temporais, objetivando, fundamentalmente, mētodos eficientes de previsão. A previsão ē um subsỉdio relevante à tomada de decisões criteriosas e como decisões criteriosas geralmente proporcionam economias considerāveis, o estu do desses mētodos se reveste de grande importância.

De um modo geral, a maioria da literatura se ocupa de mo delos tipo caixa-preta, que são ajustados à sērie histōrica segundo algum critērio pré-estabelecido. Os trabalhos sobre modelos mais vol tados à descrição interna do processo são menos frequentes. Estes modelos (que se relacionam à Teoria de Controles atravēs de uma cor respondência entre os conceitos de estado e parāmetro) possibilitam uma forma de estimação essencialmente recursiva: em cada estāgio, os parâmetros são atualizados péla estimativa anterior e pela informa ção acerca do passado suficiente para prever seu efeito no futuro:

A formulação recursiva permite uma abordagem Bayesiana ao problema de previsão. A cada estāgio pode-se prover informações,atra vēs de distribuições a priori, de duas maneiras diferentes: interna mente, estabelecendo leis de variação e propriedades estatísticas pa ra os parâmetros e externamente, refletindo a ocorrência de fatos que influenciem o comportamento futuro da sērie. E este talvez seja o ponto principal: a inclusão formal do analista, com sua experiēn cia e sensibilidade, como parte integrante do sistema de previsão.

A maioria dos mētodos de sēries temporais baseia a previ são numa anālise retrospectiva dos dados, não levando em conta sua relação com o fato presente e suas consequências futuras. Existe uma 
tendência a previsões demasiadamente calcadas no passado e este po de, em determinado instante, deixar de ser significativo. Exemplif $\underline{i}$ cando, uma série de vendas de determinado produto sofre sensivel mo dificação quando um competidor entre no mercado ou quando uma propa ganda maciça é efetuada. Os efeitos provenientes, dificeis de ex pressar quantitativamente, necessitam de distribuições de probabil $\underline{i}$ dade para descrever sua incerteza. Um modelo que opere somente com a sērie histórica, transformando mecanicamente dados de entrada em informação de saỉda, não pode prever acontecimentos desta natureza e corre o risco de, a partir de determinado instante, se tornar ina dequado. Surge então a necessidade de mētodos mais flexíveis que per mitam incorporar à sērie histōrica informações externas transmitidas pelo analista.

Baseados nestas idēias, Harrison \& Stevens (1975) desen volveram um método de previsão a curto prazo com as seguintes carac teristicas principais (além da possibilidade de prover informações a priori):

- modela a série por um modelo linear dinâmiço em que os parâmetros são atualizados atravēs de uma relação de recorrência, o filtro de Kalman, bastante conhecida em Teoria de Controles. Com isto estabe lece, implicitamente, a analogia parâmetro-estado;

- atribui aos parâmetros um significado físico (nível, inclinação, transiente), que facilita a visualização do processo e torna a pré visão uma estimação a priori;

- é aplicável quando se dispõe de um número pequeno de observações ou atē mesmo no inîcio do processo, quando ainda não se dispõe de observações;

- é capaz de detectar e se adaptar rapidamente a situações anormais como mudanças bruscas de nível, de inclinação e transiências. Isto pode ser conseguido automaticamente através de uma modelagem explị cita dessas situações ou sob intervenção direta do analista. os mé todos não Bayesianos geralmente se adaptam de um modo mais lento, produzindo erros sensiveis de previsão durante um certo tempo;

- inclui vārios modelos convencionais como regressão linear, amorte cimento exponencial e modelos lineares de sēries temporais (Box-Jen kins) como casos particulares.

Para descrever a evolução e dar consistência à idéia de estimação recursiva, seus. princípios são estabelecidos inicialmente para a regressão linear (mînimos quadrados) numa anālise baseada no trabalho de Young (1974), e posteriormente, para as sēries temporais, baseada no trabalho de Harrison \& Stevens.

\section{ALGORITMOS RECURSIVOS DE MINIMOS QUADRADOS}

0 princīpio de estimação por mínimos quadrados, devido à sua simplicidade e precisão, é uma tēcnica bastante conhecida e 
utilizada. Apōs evoluir naturalmente de um processo mecânico de ajus te para um contexto estocástico no qual as observações e os estima dores são considerados variāveis aleatărias, o método recebeu um no vo tratamento através de um trabalho de PLACKETT (1950). Nele, foi apresentada uma elegante formulação matricial recursiva que, a cada novo conjunto de observações, atualiza a estimativa dos parâmetros com um esforço computacional sensivelmente menor que o método clás sico. Apesar de toda sua potencialidade, a anālise recursiva de mî nimos quadrados não causou de imediato a devida repercussão, só vin do a se popularizar apōs um trabalho de Kalman, um engenheiro de Controles, publicado em 1961. Ele estabeleceu uma analogia entre pa râmetros e estados de um sistema, com is to pode-se generalizar a hị pótese de parāmetros constantes de Plackett e considerā-los variá veis com componentes determinīsticas e estocāsticas.

Posteriormente, em 1974, Young sintetizou todas as idéias sobre mīnimos quadrados num trabalho que objetivou estabelecer uma formulação recursiva para a anālise de sēries temporais.

\section{ABORDAGEM CLASSICA}

Seja uma variāvel $Y$ que, por hipótese, se relaciona linear mente com $N$ variāveis independentes $X_{j}, j=1, \ldots, N$ cujos valores são conhecidos. Efetua-se $k$ observações da variävel dependente $Y$, denotadas por $Y_{i}, i=1, \ldots, k$. Sendo $x_{i j}, j=1, \ldots, N \circ$ conjun to de valores associados à observação $Y_{j}$, pode-se escrever o seguin te sistema de equações:

$$
y_{i}=\theta_{1} x_{i 1}+\theta_{2} x_{i 2}+\ldots+\theta_{N} x_{i N}+v_{i} i=1, \ldots, k
$$

onde os $V_{j}$ são ruídos aleatórios com média nula, variāncia constan te $\sigma^{2}$, se realmente não correlacionados e independentes das vari $\underline{a}$ veis $x_{i 1}, x_{i 2}, \ldots, x_{i N}$ e $\theta_{j}, j=1, \ldots, N$ são os parâmetros que se deseja estimar.

o estimador de mīnimos quadrados, cuja derivação pode ser encontrada em qualquer texto sobre o assunto, tem a seguinte expres são:

$$
\theta=\left(X^{\prime} X\right)^{-1} X^{\prime} Y
$$

onde:

$$
\begin{aligned}
& \bar{\theta} \text { - estimador do vetor de parâmetros } \theta(N \times 1) \\
& X \text { - vetor de variāveis independentes }(K \times N) \\
& Y \text { - vetor de observações }(K \times l)
\end{aligned}
$$

ABORDAGEM RECURSIVA

Pode-se considerar as duas situações a seguir:

Parâmetros Constantes

Partindo da equação (1) escrita na forma 


$$
\tilde{\theta}=\left[\sum_{i=1}^{k} X^{\prime}{ }_{i} X_{i}\right]^{-1}\left[\sum_{i=1}^{k} X^{\prime}{ }_{i} Y_{i}\right]
$$

onde $x_{j} \bar{e}$ a $i$-ésima linha da matriz $x$, chega-se. apōs manipulações al gébricas ao seguinte algoritmo estocástico recursivo:

$$
\begin{aligned}
& P_{k}=P_{k-1}-P_{k-1} X^{\prime}{ }_{k}\left[\sigma^{2}+X_{k} P_{k-1} X^{\prime}{ }_{k}\right]^{-1} X_{k} P_{k-1} \\
& \hat{\theta}_{k}=\theta_{k-1}-P_{k} / \sigma^{2}\left[X^{\prime \prime}{ }_{k} X_{k} \tilde{\theta}_{k-1} X^{\prime}{ }_{k} Y_{k}\right]
\end{aligned}
$$

onde:

$$
\begin{aligned}
k= & \text { indice para indicar que a estimativa foi realizada le } \\
& \text { vando-se em conta } k \text { conjuntos amostrais }\left(X_{k}, Y_{k}\right) ; \\
P_{k}= & \text { matriz de covariância do erro da estimativa. }
\end{aligned}
$$

Parāmetros Variāveis

Na anālise anterior efetuada, considera-se implīcita a hị pótese de que os parāmetros permanecem constantes ao longo do pró cesso. Quando is to não acontece, a aplicação das fórmulas anterio res pode conduzir a erros sensīveis de estimação. Pode-se, então, utilizar dois procedimentos: o algoritmo de ponderação exponencial, que atribui peso maior às observações mais recentes e o algoritmo dinâmico estocāstico, que supõe a evolução dos parāmetros obedecen do a uma regra determinîstica superposta a uma perturbação aleatō ria. No segundo, mais flexível por permitir informações a priori se letivas sobre os parâmetros e a deteçãa das variações dos parâme tros independentemente do ruĩdo, o processo é modelado pelo seguín te sistema:

$$
\begin{aligned}
& \theta_{k}=G_{k-1, k} \theta_{k-1}+\phi_{k-1, k} W_{k-1} \\
& Y_{k}=x_{k} \theta_{k}+V_{k}
\end{aligned}
$$

onde:

$$
\begin{aligned}
& G_{k-1, k}=\text { matriz de transição }(N \times N) \\
& \phi_{k-1, k}=\text { matriz de entrada }(N \times M) \\
& W_{k-1, k}=\text { vetor de perturbações }(M \times 1) \\
& 0 \text { algoritmo de estimação é composto de duas partes: previ }
\end{aligned}
$$
são (estimação a priori), baseada na lei de variação dos parāmetros e na estimativa anterior e correção, baseada nas estimativas a prio ri e na informação contida no novo conjunto amostral. Suas fórmulas de recorrēncia são análogas ao fil tro de Kalman a ser apresentado no item subsequente. 
ANÁLISE RECURSIVA DE SERIES TEMPORAIS: METODO BAYESIANO DE PREVISAOO INTRODUÇAO

A utilização dos princīpios da estimação recursiva para a previsão de sēries temporais ē apresentada atravēs do mē todo Baye siano de Harrison \& Stevens. Apōs apontadas na introdução suas ca racterīsticas gerais, uma descrição mais profunda é efetuada neste it tem.

\section{CARACTERISTICAS DO MODELO}

Representação Paramētrica

Utiliza uma representação paramētrica do processo (em vez de uma representação funcional) que, alēm de simplificar, possibil $\underline{i}$ ta a atribuição de um sentido físico a cada parâmetro, facilitando a visualização do mecanismo evolutivo do processo e a comunicação sistema-analista. Is to porque o analista pode transmitir, atravēs de informações sỏbre os parâmetros, sua opinião acerca da influência de fatos externos e o sistema pode responder, em termos de incerte za, suas possiveis consequências.

Descrição Probabilîstica dos Parâmetros

0 vetor de parâmetros é suposto um vetor aleatōrio multi dimensional desconhecido cuja distribuição conjunta se deseja infe rir, a partir das observações, para todos os instantes. Esta infor mação é utilizada para prever os futuros valores da sērie. Deve-se ressaltar que a incerteza acerca do futuro provēm não somente da grande aleatoriedade dos fatores que o determinam mas também da in certeza acerca dos valores presentes dos parāmetros. A is to se alia a capacidade do modelo estabelecer corretamente a ligação do passa do com o futuro.

Descrição Sequencial (Recursiva)

Especifica a evolução do sistema entre dois estágios sub sequentes $(t-1, t)$ descrevendo como os parâmetros mudam no tempo, de modo sistemático e aleatōrio. Is to permite a inferência do vetor de parāmetros no instante t a partir:

- da distribuição a priori dos parāmetros no instante $(t-1)$;

- das distribuições do ruĩdo da observação e da perturba ção dos parāmetros no instante $t$;

- da observação no instante $t$.

Um aspecto importante do mētodo ē não haver exigēncia que o modelo da transição $(t-1, t)$ seja o mesmo para qualquer instante t. Is to proporciona grande flexibilidade jā que diferentes modelos podem ser apropriados a determinadas transições (por exemplo, alte rações no comportamento da sērie são esperadas como decorrência de fatos externos). 
Modelos Múltiplos

Vārios métodos estatísticos foram desenvolvidos para mani pular a incerteza dos parāmetros de um determinado modelo, mas 0 problema da incerteza quanto ao próprio modelo não recebeu o mesmo tratamento. E estranho que pouca a tenção tenha sido dada ao proble ma de haver dūvida sobre qual dos possīveis modelos está em curso em determinado instante, quando em séries temporais é bastante lóg co supor que diferentes modelos estão em curso em diferentes instan tes. Existem dois tipos principais de incerteza quanto a modelos:

- Um único modelo está em curso em todos os instantes, não sendo conhecido qual (entre um conjunto discreto de al ternativas);

- O modelo num determinado instante é uma escolha aleató ria entre um número discreto de alternativas. Is to possibilita mode lar mudanças bruscas e transiências.

MODELO LINEAR DINĀMICO

Definições

0 modelo linear dinâmico (MLD) é um sistema de equações ma triciais estocásticas especificando a relação estocástica de depen dência entre as observações e os parâmetros do processo e a evolu ção dos parâmet́tros no tempo, devido a uma regra sistemātica ineren te ao processo e a choques aleatórios.

0 modelo linear dinâmico utilizado no método é o seguinte:

Equação de observação: $Y_{t}=F_{t} \theta_{t}+V_{t}$

Equação do sistema: $\theta_{t}=\mathrm{Ge}_{t-1}+W_{t}$,

$v_{t} \simeq N\left(0, v_{t}\right)$

$w_{t}=N\left(0, w_{t}\right)$

onde:

$t$ - indice de tempo $(t=1,2,3, \ldots)$;

$Y_{t}$ - vetor de observação (Mxl) no instante $t$;

$\mathrm{F}_{\mathrm{t}}$ - matriz de variāveis independentes $(M \times N)$ conhecida no instante $t$;

$\theta_{t}$ - vetor dos parâmetros (Nxl) no instante $t$;

$G$ - matriz do sistema $(N \times N)$ conhecida;

$V_{t}$ - vetor de ruído aleatōrio (Mxl) com média nula e a ma triz de covariāncia $V_{t}$ conhecida no instante $t$;

$W_{t}$ - vetor de perturbação aleatōria (Mxl) com média nula e matriz de covariância $W_{t}$ conhecida no instante $t$.

A equação de observações específica a dependēncia estocās tica entre a observação e os parâmetros, e portanto, a distribuição de $Y_{t}$ enquanto a equação do sistema especifica a evolução dos parâa metros. 
Considerando $F_{t}$ constante, o Modelo Linear Dinâmico pode ser encarado como uma representação de espaço de estados de uma sé rie temporal em que os parâmetros admitem interpretações físicas. Is to estabelece a ligação com a teoria de controles na medida em que estimar um vetor de parâmetros pelo sistema (7) e (8) è semelhan te a estimar um vetor de estados de um sistema linear estocástico discreto a partir de um vetor de observaçöes linearmente relaciona do ao vetor de estados por uma equação matricial estocástica. 01han do desta forma, os conceitos de parāmetro e estado são equivalentes: a distinção é meramente convencional: a denominação de parāmetros é utilizada quando a variação é lenta ou não há variação e a denomina ção estado, quando a variação é rápida.

Pode-se generalizar o modelo considerando G variāvel, dis tribuição conjunta e médias não nulas para $v_{t}$ e $w_{t}$.

Estimação dos Parâmetros através do Filtro de Kalman

Para a estimação dos parâmetros do MLD é utilizada uma re lação recursiva denominada filtro de Kalman (utilizada em controles para estimar o estado de sistemas lineares dinâmicos) que atualiza, a cada instante, a informação sobre eles. Partindo da hipótese que $\theta=N\left(\hat{\theta}_{0}, P_{0}\right)$, estabelece que a distribuição a posteriori $\theta_{t} \quad \bar{e}:$ $0_{t}$

$\left(\theta_{t} \mid Y, F\right)=N\left(\bar{\theta}_{t}, P_{t}\right)$, onde a média $\hat{\theta}_{t}$ e a covariāncia $P_{t}$ são obti das pelas relaçōes:

$$
\begin{gathered}
\left.\tilde{\theta}_{t}\right|_{t-1}=G \tilde{\theta}_{t-1} \\
P_{t} t_{t-1}=G_{t-1} G^{\prime}+W_{t} \\
\tilde{\theta}_{t}=\tilde{\theta}_{t \mid t-1}+P_{t \mid t-1} F_{t}^{\prime}\left[V_{t}+F_{t} P_{t \mid t-1} F^{\prime}\right]^{-1}\left[Y_{t}-F_{t} \bar{\theta}_{t \mid t-1}\right] \\
P_{t}=P_{t \mid t-1}{ }_{t \mid t-1} F_{t}^{\prime}\left[V_{t}+F_{t} P_{t \mid t-1} F_{t}^{\prime}\right]^{-1} F_{t} P_{t \mid t-1}
\end{gathered}
$$

Assim, a distribuição a posteriori no instante $t,\left(\theta_{t} \mid y^{t}\right.$, $F^{t}$ ) pode ser calculada a partir da distribuição a priori no instan te $t-1,\left(\theta_{t-1} \mid Y^{t-1}, F^{t-1}\right)$ da observação $Y_{t}$ e das variāncias do rui do e da perturbação no instante $t, v_{t}$ e $W_{t}$. Isso possibilita, confor me aludido na introdução, uma base teōrica solida para previsões com poucas ou até mesmo nenhuma observação e o desenvolvimento de estru turas que permitem a comunicação sistema-analista.

Previsão

obtida a distribuição a posteriori do vetor de parâmetros no instante $t$, esta informação pode ser usada para inferir as dis tribuições futuras $Y_{t+k} \operatorname{com}(k=1,2, \ldots)$. As previsões decorrem da 
extrapolação de incerteza de valores presentes dos parâmetros combi nada às matrizes de covariāncia futuras $V_{t+k}$ e $W_{t+k}$, sendo portanto distribuições. Em consequência, pode-se escolher como previsão o pa râmetro mais apropriado a situação em estudo (por exemplo, o valor esperado, a mediana, a moda). Is to é importante quando as consequên cias de um erro numa direção são mais sérias que um erro da mesma magnitude no sentido oposto. Sejam:

$$
\begin{aligned}
& \vec{\theta}_{k, t}=E\left(\theta_{t+k} \mid Y^{t}, F^{t}\right) \\
& P_{k, t}=\operatorname{cov}\left(\theta_{t+k} \mid Y^{t}, F^{t}\right)
\end{aligned}
$$

A partir das estimativas (11) e (12) fornecidas pelo fil tro de Kalman, pode-se determinar (13) e (14). A previsão K passos à frente e sua matriz de covariāncia ficam

$$
\begin{aligned}
& \vec{P}_{k, t}=F_{t+k} \bar{\theta}_{k, t} \\
& \vec{P}_{k, t}=F_{t+k} P_{k, t} F^{\prime}{ }_{t+k}+V_{t+k}
\end{aligned}
$$

Quando $F_{t+k}$ não é conhecida no instante $t$, as expressões (15) e (16) se modificam para refletir a incerteza adicional na pre visão decorrente da incerteza associado ao valor de $F_{t+k}$.

MODELOS DE ESTADOS MULTIPLOS

A abordagem Bayesiana faz com que, no instante ( $t-1)$ as únicas informações necessārias para prever a observação no instante $t$, sejam a distribuição $\left(\theta_{t-1} \mid Y^{t-1}, F^{t-1}\right)$ e o MLD da transição(t-1, t). Logicamente, nada obriga a que o MLD seja o mesmo para todas as transições. Neste item, serā tratado o caso no qual nenhum MLD sozi nho descreve adequadamente o que irá acontecer ao processo no próxí mo periodo. Is to possibilita um tratamento adequado a situações anor mais que ocorram subitamente, assumindo grande importāncia se for considerado que em processo sócio-econômicos estas ocorrências são a té certo ponto normais (por exemplo em séries de vendas, observa ções esdrūxulas ocorrem com frequência, além de mudanças de nỉvel do processo em decorrēncia de propaganda ou alteração de häbitos do consumidor). Estas situações podem ser adequadamente expressas como parte de um MLD, relacionando-se variações do ruĩdo e da perturba ção aos parāmetros do processo (exceto quando as mudanças nas matrí zes $V$ e/ou $W$ não forem associadas a quantidades mensuradas a prio ri).

Para tratar este problema serão considerados modelos que em qualquer instante $t$ compreende um conjunto de MLDs cada qual com uma forma definida idēntica ( $F$ e $G$ iguais) mas diferindo entre si pela matriz de covariância do ruĩdo $V$ e perturbação $W_{t}$. 
Como ilustração, seja o modelo de crescimento linear

$$
Y_{t}=\mu_{t}+v_{t}
$$

onde:

$$
\begin{aligned}
\mu_{t} & =\mu_{t-1}+\beta_{t}+\delta \mu_{t} \\
t & =\beta_{t-1}+\delta \beta_{t}
\end{aligned}
$$

0 ruỉdo $V_{t}$ afeta o processo somente no instante $t$, não afetando os valores subsequentes. Já as perturbações do nỉvel $\delta \mu_{t}$ e inclinação $\delta \beta_{t}$ se incorporam ao processo, atuando sobre todos os valores futu ros da sērie. A Figura 1, ilustra os trēs casos, (supondo que $v_{t}$, $\delta \mu_{t}$ e $\delta \beta_{t}$ só não são nulos no instante $t$ ).
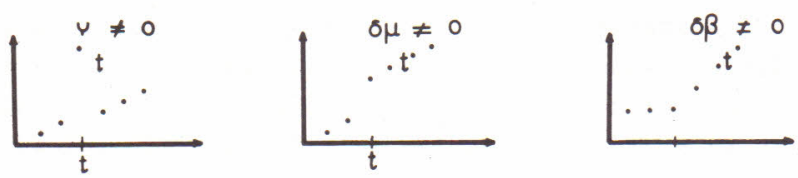

Figura 1 - Comportamento das variâncias.

Um valor muito grande de $V_{t}$ causa somente uma transiência instantānea, de $\delta \mu_{t}$ causa uma mudança de nīvel permanente e de $\delta \beta_{t}$ causa uma mudança de inclinação permanente. Pode-se, portanto, de preender o papel desempenhado pelas matrizes $V_{t}$ e $W_{t} \cdot V_{t}$ excepcio nalmente grande indica uma observação anormal no instante $t$ sem efei to no futuro; jā $W_{t}$, indica uma perturbação no instante $t$ que se incorpora ao processo. Isto permite que se modele explicitamente es tas situações supondo a existēncia de um nūmero de possīveis distri buições a partir das quais os valores de $v_{t}, \delta \mu_{t}$ e $\delta \beta_{t}$ são gerados no instante t. Como $W_{t} \bar{e}(N \times 1)$, pelo menos $(N+1)$ dessas anormalida des podem ser modeladas.

Esta colocação dá origem ao modelo de múltiplos estados. Nela um modelo $M(j)=\left(F_{t}, G, V(j)\right)$, conhecido, define um estado $j$ e caracteriza a evolução do processo no instante $t$. 0 modelo de mül tiplos estados compreende todo o conjunto $M^{(j)}$ e descreve o estado em curso no instante $t$ por um processo Markoviano. Cada um dos mode los $M^{(j)}$ tem uma probabilidade $\pi_{j}$ constante de estar em curso no ins tante t. Para exemplificar, seja.o modelo de crescimento linear:

Quatro estados bāsicos podem ser definidos:

1. Normal, 2. Mudança de Nỉvel, 3. Mudança de inclinação e 4. Tran siente.

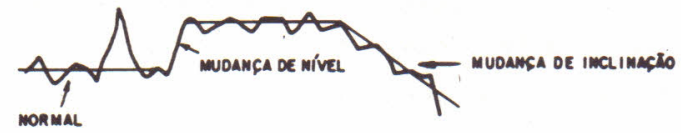

Figura 2 - Representação dos quatro estados. 
Cada um deles é caracterizado pelas variāncias do ruỉdo e da perturbação (alēm das probabilidades de estado $\pi_{j}, j=1, \ldots, 4$ ) conforme mostrado na Tabela I.

\begin{tabular}{cccc}
\hline Estado & $V_{t}$ & Var. Pert. Nivel & Var. Pert. Inclinação \\
\hline 1 & normal & muito pequena & muito pequena \\
2 & normal & grande & muito pequena \\
3 & normal & muito pequena & grande \\
4 & grande & muito pequena & muito pequena \\
\hline
\end{tabular}

Dado um modelo $M^{(j)}$, a distribuição a posteriori de $\theta_{t}$ pode ser obtida diretamente pelo filtro de Kalman usando os valores $V$ e $W$ correspondentes. Supondo que as probabilidades a posteriori de $\theta_{t}$ é uma combinação linear de $N$ distribuições normais distintas, onde $N$ é o nümero de estados. No instante $(t+1)$, a distribuição a posteriori de $\theta_{t+1}$ seria uma combinação de $N$ normais distintas. Pros seguindo, se obteria $\mathrm{N}, \mathrm{N}, \ldots$ componentes num processo proliferati vo que, de algum modo deve ser contido. O seguinte procedimento $\bar{e}$ sugerido por Harrison \& Stevens: grupar toda a informação a poste riori corresponden te ao mesmo estado $j$, preservando as informaçöes mais relevantes (e reduzindo a dimensão a posteriori $N^{2}$ para a di mensão a priori $N)$. Is to pode ser fei to pelas equações:

$$
\tilde{\theta}_{t}^{(j)}=\sum_{i=1}^{N} \operatorname{Pr}_{t}(i, j) \hat{\theta}_{t}^{(i, j)}\left[\sum_{i=1}^{N} \operatorname{Pr} t^{(i, j)}\right]
$$

$P_{t}^{(j)}=\sum_{i=1}^{N} \operatorname{Pr}_{t}^{(i, j)}\left\{p_{t}^{(i, j)}+\left[\theta_{t}^{(i, j)}-\theta^{(i, j)}-\theta_{t}^{(i, j)}\right)\left(\theta_{t}^{(i, j)}-\theta_{t}^{(i, j)}\right)\right\}$

onde: $\operatorname{Pr}{ }_{t}^{(i, j)}-\operatorname{probabilidade~de~transição~}\left(M^{(i)}, M^{(j)}\right)$ em $(t-1, t)$.

\section{MODELOS BAYESIANOS COM DESCONTOS}

A formulação do modelo Bayesiano apresentado anteriormen te apesar de simples e elegante, apresenta como dificuldade o conhe cimento prévio das variāncias dos ruỉdos ( $V_{\varepsilon}, V_{\mu}$ e $V_{\beta}$ no caso do $M(L)$, o que dificulta ao usuārio sem maiores conhecimentos estatīs ticos. Na tentativa de contornar esta dificuldade Ameen \& Harrison (1983), apresentaram a formulação do MLD com descontos. Neste traba Iho vamos descrever o comportamento do Modelo de Crescimento Linear de Estados Mültiplos com Descontos.

MCL DE ESTADOS MOLTIPLOS COM DESCONTOS

Neste trabalho vamos considerar a caracterização de quatro 
estados possiveis, tais como:

a. Estado 1: Normal

E o estado padrão, em que o comportamento da sērie não apresenta qualquer tipo de descontinuidade sendo as variaçoes obser vadas causadas basicamente pela variāncia $V_{t}$;

$$
M_{t}^{(1)}=\left\{\underline{F}_{t}, \underline{G}, V_{t}, \underline{B}\right\}
$$

b. Estado 2: Transiente

E o estado em que ocorrem descontinuidades bruscas na sé rie, sendo porém de carāter transitōrio. Este comportamento è explí cado por um valor acentuado de variāncia do ruido das observaçóes;

$$
M_{t}^{(2)}=\left\{\underline{F}_{t}, \underline{G}, V_{G}, \underline{B}\right\}
$$

onde $V_{G} \gg V_{t}$ (Usualmente $V_{G}=\alpha V_{t}^{(N)}$ )

c. Estado 3: Mudanca de Nivez

Adequado para a modelagem de mudanças estruturais no pro cesso gerador da série, que acarreta numa mudança permanente no nī vel da série. Este comportamento é explicado por um valor pequeno do fator de desconto do nível;

$$
M_{t}^{(3)}=\left\{\underline{F}, \underline{G}, V_{t}, \underline{B}^{(N)}\right\}
$$

onde $\underline{B}=\operatorname{diag}\left\{B_{\mu}, \beta_{\beta}\right\}$, sendo $\beta_{\mu}^{(N)}=\left[B_{\mu}^{(N)} I^{k 3} ; k 3>>1\right.$

a. Estado 4: Mudanga de Inclinação

E aplicado à taxa de crescimento da série $\beta_{t}$, é represen tado por:

$$
M_{t}^{(4)}=\left\{\underline{F}_{t}, \underline{G}, V_{t}, \underline{B}^{(I)}\right\}
$$

onde $\underline{B}^{(I)}=\operatorname{diag}\left\{\beta_{\mu}^{(N)} ; \beta_{\beta}^{(I)} ; \beta_{\beta}^{(I)}\right\}=\left\{\beta_{\beta}^{(N)}\right\}^{k 4} ; k 4 \gg 1$

Vemos portanto que a caracterização dos quatro estados do MCL com descontos é feita a partir do conhecimento prévio de 6 quan tidades: os valores nominais $V_{t}^{(N)} ; \beta(N)$ e $\beta{ }_{\beta}^{(N)}$ e os fatores $\alpha, k 3$ e k4.

\section{APL ICAÇÃO}

0 método descrito foi aplicado à uma sērie temporal simu 1 ada de 20 observações geradas de uma população Normal $(\mu=1 ; \sigma=0.1)$ e nela foi introduzido as seguintes descontinuidades:

i) Transiente de $5 \sigma$ em $t=5$; 
ii) Mudança de Nivel Permanente de 2.5o em $t=10$;

iii) Mudança de Inclinação Permanente de $2.5 \sigma$ em $t=15$.

A tabela com os valores da série e o respectivo gräfico es tão apresentados na Tabela I e a Figura 1, respectivamente.

TABELA I - VALORES DA SERIE SIMULADA

\begin{tabular}{cc}
\hline \hline No da Observação & Valor \\
\hline 1 & 1.11 \\
2 & 1.14 \\
3 & 1.11 \\
4 & 1.10 \\
5 & 1.75 \\
6 & 1.12 \\
7 & 1.11 \\
8 & 1.12 \\
9 & 1.19 \\
10 & 1.54 \\
11 & 1.49 \\
12 & 1.51 \\
13 & 1.53 \\
14 & 1.55 \\
15 & 1.10 \\
16 & 1.03 \\
17 & 1.02 \\
18 & 1.03 \\
19 & 0.99 \\
20 & 0.84 \\
\hline
\end{tabular}

1.750

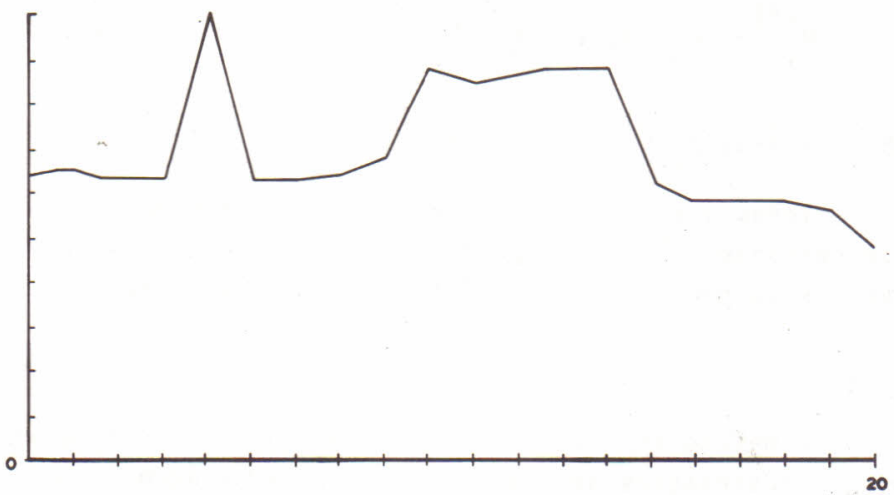

Figura 1 - Grāfico da Série Simulada com 20 observações. 
ANĀL ISE

Procedemos inicialmente à calibração dos hiperparāmetros do modelo, apōs vārias tentativas, optamos por adotar os próprios valores adotados no programa SBPC, que são:

$$
\begin{array}{llll}
\pi_{1}=0.8000 & \pi_{2}=0.0940 & \pi_{3}=0.0830 & \pi_{4}=0.0230 \\
\beta_{2}=0.90 & B_{2}=0.95 & k 3=15 & k 4=15 \\
\alpha=90 \quad v_{t}=0.001 &
\end{array}
$$

As probabilidades condicionadas estão apresentadas na Ta bela II.

TABELA II - PROBABILIDADES CONDICIONADAS

\begin{tabular}{ccccc}
\hline$M^{(1)}$ & $M^{(2)}$ & $M(3)$ & $M(4)$ & $\begin{array}{c}\text { Modelo de } \\
\text { maior } \\
\text { probab.i1idade }\end{array}$ \\
\hline 0.8789 & $t$ & $t$ & $t$ & 1 \\
0.9045 & 0.0467 & 0.0491 & 0.0253 & 1 \\
0.8689 & 0.0323 & 0.0763 & 0.0226 & 1 \\
0.8977 & 0.0172 & 0.0608 & 0.0243 & 1 \\
0.1327 & 0.8158 & 0.0450 & 0.0064 & 2 \\
0.9865 & 0.0123 & 0.0012 & 0.0000 & 1 \\
0.9871 & 0.0123 & 0.0006 & 0.0000 & 1 \\
0.9766 & 0.0124 & 0.0110 & 0.0000 & 1 \\
0.9395 & 0.0139 & 0.0466 & 0.0000 & 1 \\
0.3117 & 0.1361 & 0.3900 & 0.1623 & 1 \\
0.9114 & 0.0117 & 0.0556 & 0.0213 & 1 \\
0.9198 & 0.0122 & 0.0478 & 0.0202 & 1 \\
0.9183 & 0.0129 & 0.0485 & 0.0204 & 1 \\
0.9138 & 0.0135 & 0.0514 & 0.0213 & 1 \\
0.1138 & 0.3322 & 0.4858 & 0.0682 & 1 \\
0.8802 & 0.0187 & 0.0766 & 0.0246 & 1 \\
0.8625 & 0.0209 & 0.0846 & 0.0320 & 1 \\
0.8612 & 0.0205 & 0.0833 & 0.0350 & 1 \\
0.8401 & 0.0238 & 0.0954 & 0.0407 & 1 \\
0.6538 & 0.0554 & 0.2045 & 0.0863 & 1 \\
\hline
\end{tabular}

A adaptabilidade (automātica) do modelo pode ser sentida em:

a) em $t=5$, que indica um transiente;

b) em $t=10$, que indica uma mudança de nîvel;

c) em $t=15$, o modelo interpreta como uma uma mudança de nível, mas na realidade o que ocorreu foi uma mudança de inclinação. 
Assim, a perfformance do modelo possivelmente pode ser me lhorada, pois o sistema foi insensīvel a mudança de inclinação (e tado 4) ocorrida no periodo 15.

CONCLUSÃO

Neste trabalho pode-se verificar que a modelagem Bayesia na é realmente muito útil para a anālise de sēries que apresentam mudanças estruturais, bem como a presença de outlier, pois permite ao investigador a incorporar essas descontinuidades ao modelo em es tudo.

Quanto ao programa utilizado pode-se afirmar que realmen te está muito bem elaborado, pois somente quanto a mudança de incl $\underline{i}$ nação é que o modelo não apresentou sensibilidade instantânea, mas analisando-se mais algumas observações que foram extrapoladas na sé rie, pode-se afirmar que o sistema leva em média 7 observações para detectar uma mudança de inclinação.

\section{B I BL I OGRAF I A}

1. FARIAS NETO, J.J. (1981). Modelo Bayesiano de Crescimento Linear Aplicado à Previsão de Sēries Temporais. Tese de Mestrado, Gru po de Sistemas, DEE PUC-Rio.

2. SoUZA, R.C. (1988). Modelos Bayesianos para Previsão e Controle Via CUSUM. Minicurso apresentado na XIRRE, IERMQ e III ERERS, Santa Maria, RS.

3. Anotações de Aula do Prof. Reinaldo Castro Souza. PUC. Rio.

Recebido em dezembro, 1989; aceito em janeiro, 1990. 